\title{
Design Considerations in An Active Matched Semiloop Array with Non-Foster Networks
}

\author{
Fernando Albarracín-Vargas, Daniel Segovia-Vargas \\ Department of Signal Theory and Communications \\ University Carlos III of Madrid \\ Av. de la Universidad 30, 28911, Leganés, Madrid, Spain. \\ falbarracin@tsc.uc3m.es, dani@tsc.uc3m.es
}

\begin{abstract}
Most of the work in phased arrays loaded with nonFoster forms has been made about characteristics that look for avoiding beam squint in radiation along frequency or enhancing the array beam-width or gain at a broad frequency range. This work aims at pointing some considerations about a two elements array loaded with non-foster forms in terms of impedance matching and radiation pattern.
\end{abstract}

Keywords—small antennas; phased arrays; non-Foster networks, sensitivity

\section{INTRODUCTION}

Active loaded phased array has been designed by some authors in such a way the objective consist of increasing gain over a broadband, given continuity of current at lower frequencies, or improving the scan element pattern (SEP) using passive and active reactive elements, well connected between the array elements (coupling configuration) well connected at each element input port (matching configuration) [1]. Those reactive elements would be lumped inductors or capacitors in the passive case, and negative engineered capacitors or inductors realized by means of the negative impedance converters (NIC) for the active ones. A NIC is a two port network where one port presents a negated version of the impedance loading the other port, so elements that do not obey the Foster's reactance theory [2] can be implemented.

Among other work in phased arrays loaded with active elements, it is possible to find the inclusion of negative group delay networks (NGD) in the feeding of a linear array [3] or a parasitic element loaded with a NIC [4]. Both cases take advantage of the property of increasing phase-response (i.e. another non-Foster behavior) of the NICs and NGD with frequency, looking for a broadband squint-free and steerable pattern. On the other hand, when the intended frequency band is such as the electrical size of the elements in the array is around $0.1 \lambda$, the designer have to deal with an additional constraint: high reactance value and a strongly frequency dependent resistance presented by electrically small antennas (ESA), related with the well-known high quality factor $Q$ in this structures [5], that implies hard working in broadband impedance matching [6].

\author{
Vicente Gonzalez-Posadas \\ Department of Audiovisuals and Communications \\ Polytechnic University of Madrid \\ Ctra. de Valencia Km 7, 28031, Madrid, Spain. \\ vgonzalzapdiac.upm.es
}

In this work, a two-element linear array composed of two semiloops, as it is shown in Fig. 1, connected through a nonFoster matching network ( $\mathrm{MN})$ in a coupling configuration, is presented as a comparative design between two cases: an ideal non-Foster MN (a series negative inductor, $L<0$ ) and a MOSFET based NIC acting as the realized active MN. A FR4 slab, with $\varepsilon_{\mathrm{r}}=4.3$ and $1.5 \mathrm{~mm}$ in thickness, contains the coplanar array elements. The design aims at matching the array at the lower part of the VHF band (under $150 \mathrm{MHz}$ ). The natural frequency for the array, $\left(2 \pi R=\lambda_{0}\right)$ is $1200 \mathrm{MHz}$, where the typical double lobe (i.e. along the $+\mathrm{z}$ and $-\mathrm{z}$ axis) is observed for a $\lambda_{0} / 2$ separation between elements.

\section{ACTIVE IMPEDANCE MATCHING DESIGN}

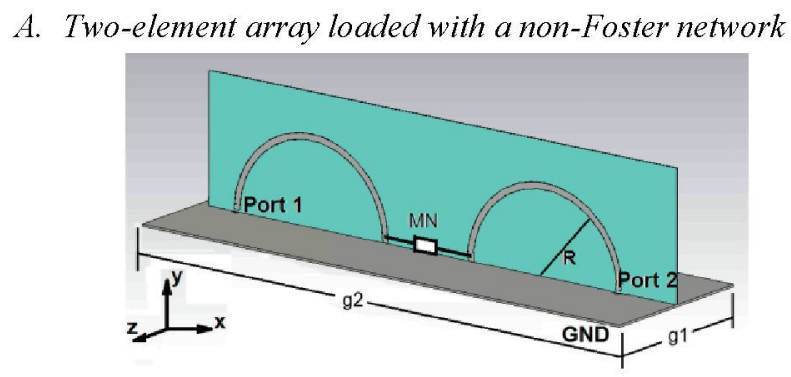

Fig. 1. Sketch of the two elements array. All dimensions in mm: $R=40$, $\mathrm{g}_{1}=80, \mathrm{~g}_{2}=260$.

A multiport antenna approach is used to deduce the analytical impedance, $Z_{M N}^{a n}$, that have to be implemented well with an ideal non-Foster $\mathrm{MN}$ well with a transistorized circuit for broadband impedance matching. As a previous work in [7], the authors found the most suitable location for an active MN in a single semiloop. Such location is the opposite side from the input port. Due to the symetry of the two-element array here (see Fig. 1), it is possible to deduce the analytical $Z_{M N}^{a n}$ by using a $S$-parameter matrix of a two-port structure extracted from any fullwave simulator (port 1 and port $\mathrm{MN}$ ); the other port of the array is terminated with the system impedance: $50 \Omega$. Then, we can do some calculus with the input reflection coefficient, $\Gamma_{N}$ given by (1).

$$
\Gamma_{I N}=S_{11}+\frac{S_{12} \cdot S_{21} \cdot \Gamma_{M N}}{1-S_{22} \cdot \Gamma_{M N}}=0 \rightarrow
$$




$$
\rightarrow \Gamma_{M N}^{a n}=\frac{S_{11}}{S_{22} \cdot S_{11}-S_{12} \cdot S_{21}}
$$

Once (1) is set to zero, the optimum reflection coefficient that the active $M N$ must provide, $\Gamma_{M N}^{a n}$, can be extracted as (2) and the associated non-Foster impedance, $Z_{N I C}^{a n}$ needed to match the antenna at port 1 and 2 can be expressed as (3).

$$
Z_{M N}^{a n}=Z_{0} \cdot\left(\frac{S_{22} \cdot S_{11}-S_{12} \cdot S_{21}+S_{11}}{S_{22} \cdot S_{11}-S_{12} \cdot S_{21}-S_{11}}\right)
$$

\section{B. Sensitivity analysis over the array structure:}

When this impedance: $Z_{M N}^{a n}$ is placed between the semiloops, the reflection coefficient at port 1 and $2, \Gamma_{N N}$, is ideally equal to 0 in the design band. Here it is possible to use the sensitivity parameter Sens, introduced by the authors in [7] and derived from (4) and (5) in order to understand how the changes in the $\mathrm{MN}$ impedance, affects the input impedance in the array ports.

$$
\begin{gathered}
\Delta \Gamma_{I N}=\left.\frac{\partial \Gamma_{I N}}{\partial \Gamma_{N I C}}\right|_{\Gamma_{N I C}=\Gamma_{N I C}^{a n}} \cdot \Delta \Gamma_{N I C}=\operatorname{Sens} \cdot \Delta \Gamma_{N I C} \\
\text { Sens } \\
\text { Sens }=\left|\frac{\left(S_{11} \cdot S_{22}-S_{21} \cdot S_{12}\right)^{2}}{S_{21} \cdot S_{12}}\right|
\end{gathered}
$$

Figure 2 shows the parameter Sens with frequency for the proposed two-element array compared to the single two-port semiloop. Values near $10 \mathrm{~dB}$ or lower can be treated as low sensitivity. Sens parameter enlarges in the lower VHF band, but still remains under reasonable values for broadband impedance matching.

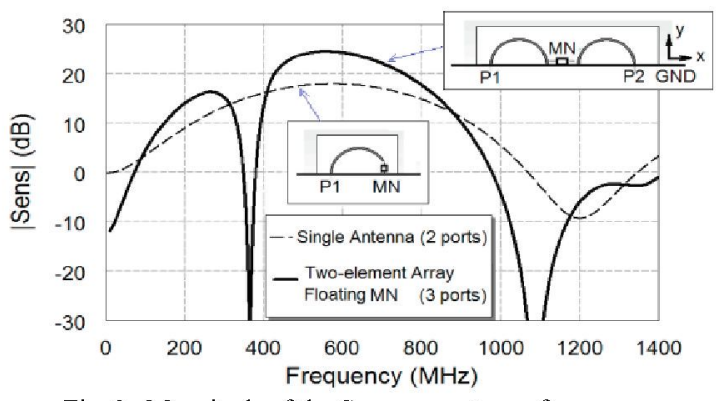

Fig. 2. Magnitude of the Sens parameter vs frequency.

Two different networks are connected as a $\mathrm{MN}$ between the array elements. The first one, a series non-Foster ideal inductor (inset in Fig. 3), presents an important impedance matching response, compared to the transistor based NIC (BF998). This fact confirms the need of a simple non-Foster structure, even though it is an idealized case. It is worth to note that the maximum frequency at which the NIC acts as an active $\mathrm{MN}$ is expected to be $180 \mathrm{MHz}$. That frequency corresponds with the upper bound of the lower band obtained $\left(S_{11}<-6 \mathrm{~dB}\right)$, for the NIC case, depicted in Fig. 3.

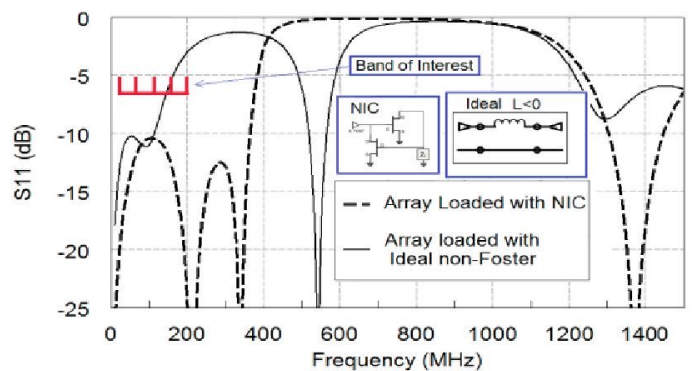

Fig. 3. $S_{11}$ and $S_{22}$ response of the array for both cases: Ideal and realized non-Foster MN.

\section{RADIATION PERFORMANCE}

In terms of radiation performance, it was found an omnidirectional response in the horizontal plane, in the lower VHF band (100 MHz) for the NIC loading the array, as can be seen in Fig. 4(a). In $1200 \mathrm{MHz}$ (see Fig. 4(b)) the obtained pattern remains basically unchanged, compared with the unloaded array when two in-phase signals are applied at the input of each element. For the series negative inductor case, the radiation response in both frequencies is not reported here, being almost the same as the one with a transistorized MN.

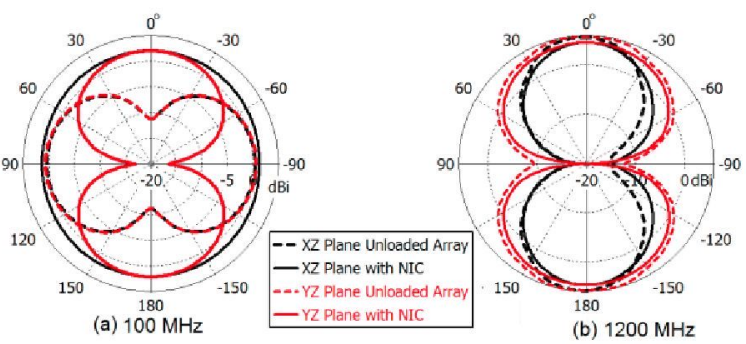

Fig. 4. Directivity pattern of the active loaded Array.

As a conclusion it is possible to state an important advantage in loading symmetric structures with active $\mathrm{MN}$. Two-element semiloop array appears to be a suitable choice for broadband impedance matching. However, the broadband characteristic is constrained by the NIC maximum frequency. At the time of the conference, the authors hope to have measured results.

\section{REFERENCES}

[1] R. Hansen, "Dipole arrays with non-foster circuits," in IEEE International Symposium on Phased Array Systems and Technology, 2003, 2003, pp. 40-44.

[2] R. M. Foster, "A reactance theorem," Bell Syst. Tech. J., vol. 3, no. 2, pp. 259-267, Apr. 1924.

[3] H. Mirzaei and G. V. Eleftheriades, "Arbitrary-angle squint-free beamforming in series-fed antenna arrays using non-Foster elements synthesized by negative-group-delay networks," IEEE Trans. Antennas Propag., vol. 63, no. 5, pp. 1997-2010, May 2015.

[4] M. M. Jacob, J. Long, and D. F. Sievenpiper, "Non-Foster loaded parasitic array for broadband steerable patterns," IEEE Trans. Antennas Propag., vol. 62, no. 12, pp. 6081-6090, Dec. 2014.

[5] S. R. Best, "Low Q electrically small linear and elliptical polarized spherical dipole antennas," IEEE Trans. Antennas Propag., vol. 53, no. 3, pp. 1047-1053, Mar. 2005.

[6] L. J. Chu, "Physical limitations of omni-directional antennas," J. Appl Phys., vol. 19, no. 12, pp. 1163-1175, Dec. 1948.

[7] F. Albarracin-Vargas, E. Ugarte-Munoz, V. Gonzalez-Posadas, and D. Segovia-Vargas, "A design strategy of active matched small-antennas with non-Foster elements," in 2015 9th European Conference on Antennas and Propagation (EuCAP), 2015, pp. 1-4. 